\title{
Rotating Parabolic-Reflector Antenna Target in SAR Data: Model, Characteristics, and Parameter Estimation
}

\author{
Bin Deng, Hong-Qiang Wang, Yu-Liang Qin, Sha Zhu, and Xiang Li \\ College of Electronic Science and Engineering, National University of Defense Technology, China \\ Correspondence should be addressed to Bin Deng; sagitdeng@163.com
}

Received 22 October 2012; Accepted 3 March 2013

Academic Editor: Gui Gao

Copyright (C) 2013 Bin Deng et al. This is an open access article distributed under the Creative Commons Attribution License, which permits unrestricted use, distribution, and reproduction in any medium, provided the original work is properly cited.

\begin{abstract}
Parabolic-reflector antennas (PRAs), usually possessing rotation, are a particular type of targets of potential interest to the synthetic aperture radar (SAR) community. This paper is aimed to investigate PRA's scattering characteristics and then to extract PRA's parameters from SAR returns, for supporting image interpretation and target recognition. We at first obtain both closed-form and numeric solutions to PRA's backscattering by geometrical optics (GO), physical optics, and graphical electromagnetic computation, respectively. Based on the GO solution, a migratory scattering center model is at first presented for representing the movement of the specular point with aspect angle, and then a hybrid model, named the migratory/micromotion scattering center (MMSC) model, is proposed for characterizing a rotating PRA in the SAR geometry, which incorporates PRA's rotation into its migratory scattering center model. Additionally, we in detail analyze PRA's radar characteristics on radar cross-section, high-resolution range profiles, time-frequency distribution, and 2D images, which also confirm the models proposed. A maximal likelihood estimator is developed for jointly solving the MMSC model for PRA's multiple parameters by optimization. By exploiting the aforementioned characteristics, the coarse parameter estimation guarantees convergency upon global minima. The signatures recovered can be favorably utilized for SAR image interpretation and target recognition.
\end{abstract}

\section{Introduction}

Parabolic-reflector antennas (PRAs), due to their excellent beam directionality, are widely used in radar surveillance, navigation, microwave communication, and radio astronomy. Although they are antennas in themselves, PRAs are also present as a particular type of targets, gaining growing interest in some scenarios to the synthetic aperture radar (SAR) community [1]. Their scattering and rotation characteristics, which are obtainable with SAR, may be of valuable aid for target recognition. Therefore, it is desirable to detect and recognize them from SAR returns or imagery. However, this work is difficult because of two reasons. On one hand, the backscattering of PRAs is so complicated that they cannot be characterized by point scattering centers, and the directionality, favorable as it is for PRAs as antennas, results in aspect sensitivity and weak backscattering at most aspect angles when they are present as SAR targets. On the other hand, their rotation, due to mechanical scanning, changes the Doppler history and therefore makes their SAR images blurred and enigmatic. And unfortunately, rotation cannot be processed by the mature technique, that is, SAR ground moving target indication (SAR/GMTI), though it is a valid tool for uniformly moving targets [2].

Conventionally, rotating PRAs (RPRAs) were investigated as micromotion targets. In 2009, the SAR/micromotion target indication concept was presented to generalize the SAR/GMTI technique and to intensively examine the detection and imaging problems for micromotion targets [3]. Actually, early in 2004, the picket fence shape was revealed to be representative of a rotating radar dish in the SAR azimuth image [4], but the dish (viz. PRA) was simplified as a combination of point scatterers, which are not qualified for characterizing PRA's complex scattering. In 2005 and 2010, wavelet decomposition and chirplet decomposition were used to successfully separate the return of an RPRA from that of stationary parts. The returns were collected by the US Navy APY-6 radar [5, 6]. After separation, time-frequency distribution (TFD) was utilized to analyze the phase modulation and autocorrelation to estimate the rotating frequency. 
The RPRA detected was annotated in the SAR image [1]. However, the RPRA's signature blurred in SAR imagery was not explained, and the aforementioned approaches are susceptible to range cell migration (RCM). TFD also usually suffers from cross-term interference. In 2007, RPRA data, collected by a microwave SAR system in University of Zurich, were analyzed [7]. A point-symmetrical image of the RPRA was obtained, and the time-frequency image showed a small fraction of a sinusoidal phase modulation. The conclusions, however, are only valid for slow rotating frequency and short coherent processing interval (CPI). PRA's radar characteristics have not been deeply studied either.

In this paper, we focus on characterization and non-TFDbased estimation of PRAs, both stationary and rotating, in spotlight SAR data. There are different types of PRAs, but we restrict our scope to a rotation-symmetrical PRA with a single reflector. Instead of processing returns in a single range cell, we use the phase history in the wavenumber domain, that is, the 2D Fourier transform (FT) of complex imagery, to extract PRAs and their parameters. We build for a PRA a new non-point-scattering center model able to describe the aspect sensitivity and the sliding of scatterers and then incorporate the motion model into it to obtain a hybrid scattering/motion model. Radar characteristics of PRAs are then analyzed. Afterwards, an estimation algorithm for this model is proposed. This idea fully exploits a priori information on target models, implements joint estimation of scattering and motion parameters, and circumvents TFD and other tough issues, such as clutter suppression, detection, RCM correction, and parameter estimation which have to be conducted separately and explicitly in SAR/GMTI.

This paper is organized as follows. In Section 2, geometrical optics (GO), physical optics (PO), and graphical electromagnetic computation (GRECO) are used to calculate the backscattering or complex radar cross-section (RCS) of a PRA. Then, based on the calculated results, we propose a new scattering center model and a hybrid scattering/motion model in Section 3. We then in Section 4 analyze the characteristics of PRAs. A few interesting signatures of PRAs are revealed, for example, periodicity of high-resolution range profile (HRRP) sequences, bowl- and bowknot-shaped images. Sections 5 and 6 provide a maximum likelihood estimator and a few results, and conclusions are reported in Section 7. The derivation throughout the paper may be a little difficult to follow, and we will try our best to clarify the problem.

\section{Complex RCS Calculation for PRA}

Backscattering of an antenna consists of two parts, that is, structured scattering, whose scattering principle is the same of as common objects, and pattern scattering, which is closely related to antenna's loads. Pattern scattering is weak for a PRA and, hence, not taken into account here. We will calculate PRA's complex RCS via GO, PO, and GRECO. GO is simple and is particularly suitable for smooth surface. PO has high accuracy near target's normal direction and can provide closed-form solutions in some particular aspects. And GRECO, as a tool and software, incorporates many

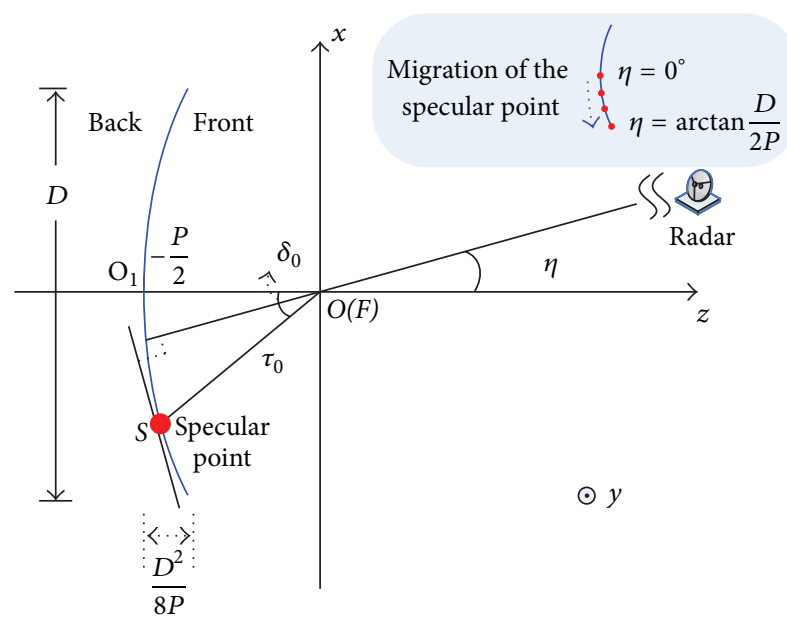

FIGURE 1: PRA geometry ( $x-z$ slice).

electromagnetic methods and can efficiently provide highprecision numerical solutions.

2.1. GO. As Figure 1 shows, let $P$ denote the focus parameter of a PRA, $\tau$, and $\delta$ denote the radius and angle (relative to $-z$ axis) of any point on a PRA. Other parameters encountered are defined in Figure 1 where the focus is located at the origin. $\mathrm{O}-\mathrm{z}$ is the symmetrical axis. Then, the amplitude of the PRA's complex RCS, that is, the square root of RCS, can be obtained from the GO theory [8]

$$
\left|\sqrt{\sigma_{\mathrm{GO}}}\right|=\frac{\sqrt{\pi} P}{\cos ^{2} \eta} .
$$

Its phase can be easily derived from the position of the specular point $S$. Combining the amplitude and phase, we have the complex RCS

$$
\sqrt{\sigma_{\mathrm{GO}}}=\frac{\sqrt{\pi} P}{\cos ^{2} \eta} \exp \left(-j K \frac{P}{2 \cos \eta}\right),
$$

where $K=4 \pi f / c$ is the wavenumber, $c$ is the speed of light, and $f$ is the frequency. When choosing PRA's bottom center $\mathrm{O}_{1}$ as the phase reference, we need to multiply (2) with $\exp (j K \cdot P / 2 \cdot \cos \eta)$ and then have

$$
\sqrt{\sigma_{\mathrm{GO}}}=\frac{\sqrt{\pi} P}{\cos ^{2} \eta} \exp \left(-j K \frac{P \sin ^{2} \eta}{2 \cos \eta}\right) .
$$

Equations (2) and (3) hold only when $\eta$ is chosen such that the specular point does not move outside the PRA. Specifically, when the incident field illuminates the PRA front side, $\eta$ should be subjected to

$$
|\eta| \leq \arctan \frac{D}{2 P}
$$

2.2. $P O$. To facilitate application of $\mathrm{PO}$, we use the Cartesian coordinate system and the cylinder coordinate system. Then in Figure 1, for any point $Q$ in the PRA, suppose that its 
position in the cylindrical coordinate system is $(\rho, \varphi, z)=$ $\left(\rho, \varphi, \rho^{2} /(2 P)-P / 2\right)$. It can be vectorized in the Cartesian coordinate system as

$$
\mathbf{r}^{\prime}=(x, y, z)^{T}=\left(\rho \cos \varphi, \rho \sin \varphi, \frac{\rho^{2}}{2 P}-\frac{P}{2}\right)^{T} .
$$

The normal vector at $Q$ is

$$
\widehat{\mathbf{n}}=\left(-\frac{\rho \cos \varphi}{P},-\frac{\rho \sin \varphi}{P}, 1\right)^{T} / \sqrt{1+\frac{\rho^{2}}{P^{2}}} .
$$

The backscattering direction can be expressed by

$$
\widehat{\mathbf{r}}=(\sin \eta, 0, \cos \eta)^{T} \text {. }
$$

According to PO, the complex RCS of the PRA can be written as

$$
\sqrt{\sigma_{\mathrm{PO}}}=-j \frac{k}{\sqrt{\pi}} \int_{0}^{2 \pi} \int_{0}^{D / 2} \widehat{\mathbf{r}} \cdot \widehat{\mathbf{n}} \exp \left(j K \widehat{\mathbf{r}} \cdot \mathbf{r}^{\prime}\right) \sqrt{1+\frac{\rho^{2}}{P^{2}}} \rho d \rho d \varphi .
$$

Generally, (8) can be calculated by using numerical methods, and its closed-form solution cannot be obtained due to its complexity. However, the closed-form solution exists for some particular incident angles. When $\eta=0$, for example, the complex RCS we derive is

$$
\begin{aligned}
\left.\sqrt{\sigma_{\mathrm{PO}}}\right|_{\eta=0} \\
=-j 2 \sqrt{\pi} P \sin \left(K \frac{D^{2}}{16 P}\right) \exp \left[-j K\left(\frac{P}{2}-\frac{D^{2}}{16 P}\right)\right] \\
=\sqrt{\pi} P\left[\exp \left(-j K \frac{D^{2}}{16 P}\right)-\exp \left(j K \frac{D^{2}}{16 P}\right)\right] \\
\quad \times \exp \left[-j K\left(\frac{P}{2}-\frac{D^{2}}{16 P}\right)\right] .
\end{aligned}
$$

2.3. GRECO. GRECO is an effective tool for calculating (complex) RCS of electrically large objects [9]. It is capable of calculating the reflected and diffracted fields with high precision and efficiency by using graphic hardware acceleration and can also perform well for a wide range of aspect angles. Therefore, we select it as a numeric method and benchmark for the GO and PO methods. The result will be given in Section 6 .

\section{Scattering Center Model and Hybrid Model}

3.1. Migratory Scattering Model for a Stationary PRA. The scattering center models have developed from the point scattering model, damped exponential/Prony model [10], through the geometric theory of diffraction (GTD) model [11], localized scattering model [12], and attributed scattering model [13], to the polynomial model [14] and migratory scattering model [15]. Their difference lies in that they use different models to characterize the dependency of complex RCS, including amplitude and/or phase, on frequency and/or aspect angle. We can find from (2) that, for a PRA, the complex RCS amplitude depends on aspect angle $\theta(\theta=\eta$ when aspect angle is relative to PRA's axis of symmetry), resulting in aspect sensitivity, while the phase also depends on aspect angle but the dependency is not of the form of $\sin \theta$ or $\cos \theta$ as ideal point scattering is $(1 / \cos \eta$ instead), which means that the scattering center will move when the aspect angle varies. By (2), we have in effect proven that a PRA can be approximated by the migratory scattering model containing a single scattering center, and the approximation has sound physical grounds. However, the amplitude term of complex RCS in (2), enough as it is for coarse analysis, is not an accurate model intended for parameter estimation, since GO does not take into account more scattering components, for example, edge diffraction. Therefore, we use a function, $s\left(\eta_{\theta}\right)$, to describe arbitrary aspect dependency. Then, from (3), we obtain the migratory scattering model for a stationary PRA

$$
g(K, \theta)=s(\theta) \exp \left[-j K\left(x \cos \theta+y \sin \theta+\frac{P \sin ^{2} \eta_{\theta}}{2 \cos \eta_{\theta}}\right)\right],
$$

where $(x, y)$ are the coordinates of the PRA's bottom center $\mathrm{O}_{1}$, and $\eta_{\theta}$ is the $\theta$-dependent incident angle.

For instance, When choosing PRA's geometrical center as the phase reference (in order for agreement with the GRECO geometry in Figure 3 and Section 4), we have

$$
\begin{aligned}
g(K, & \left.\theta^{\prime}\right) \\
& =s\left(\theta^{\prime}\right) \exp \left[-j K\left(\frac{\sin \theta^{\prime}}{4}+\frac{P \sin ^{2}\left(\pi / 2-\theta^{\prime}\right)}{2 \cos \left(\pi / 2-\theta^{\prime}\right)}\right)\right] \\
& =s\left(\theta^{\prime}\right) \exp \left[-j K\left(\frac{\sin \theta^{\prime}}{4}+\frac{P \cos ^{2} \theta^{\prime}}{2 \sin \theta^{\prime}}\right)\right] .
\end{aligned}
$$

When $\theta^{\prime}=\omega t$ is around $90^{\circ}$ (PRA's front against radar), where $\omega$ is the angular frequency and $t$ is the slow time, the instantaneous Doppler can be obtained by taking the derivative of the phase in (11)

$$
f_{d} \approx \frac{17.5}{\lambda} \cos (\omega t) \approx \frac{8.75 \pi}{\lambda}-\frac{17.5 \omega}{\lambda} t
$$

Equation (12) indicates that the PRA's return in azimuth is a sinusoidal frequency-modulated (SFM) signal or approximately a linear frequency-modulated (LFM) signal when the PRA is illuminated broadside.

3.2. Hybrid Model for a RPRA in the SAR Geometry. It is well known that a PRA usually possesses rotation in operation. Thus, we need to establish its motion model by finding the range from SAR to the specular point $S$. For the SAR geometry shown in Figure 2, $O$ is the center of the scenario 


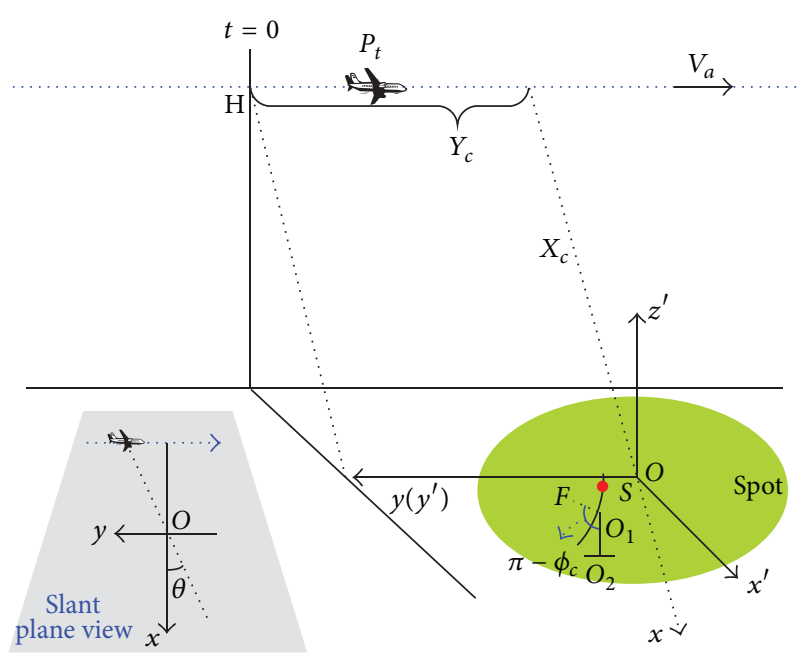

FIGURE 2: SAR geometry with a RPRA.

illuminated by SAR. Assume that a PRA is rotating around its vertical axis, and the pivot is the top of the vertical axis, identical to $O_{1}$. The angle between the $O-z^{\prime}$ axis and the PRA's axis of symmetry, $\phi_{c}$, remains constant during the rotation around $\mathrm{O}_{1} \mathrm{O}_{2} \cdot \theta_{c}=-\arctan \left(Y_{c} / X_{c}\right)$ is the slant angle. When $Y_{c}=0$, SAR is side-looking, and otherwise it operates in squint-looking mode. $\theta$ is the angle of light of sight (LOS) in $O-x y$ (i.e., slant plane). The relationship between $\theta$ and $t$ is

$$
X_{c} \tan \theta=V_{a} t-Y_{c}
$$

When $\theta_{c}=0$ and the extent of aspect angle $\theta$ is small, $\tan \theta \approx \theta$, and then (13) reduces to

$$
\theta=\frac{V_{a}}{X_{c}} t
$$

Clearly, $\theta$ also has the meaning of slow time.

For deriving the range model, we at first make the following definitions.

In $O-x y$ at slow time $t$,

$$
\begin{aligned}
& P_{t} \text { is at }\left(-X_{c}, Y_{c}-V_{a} t\right) \text { (SAR's position), } \\
& O_{1} \text { is at }(x, y) \text { (bottom center of the PRA). }
\end{aligned}
$$

In $O-x^{\prime} y^{\prime} z^{\prime}$ at slow time $t$, the normalized vectors of $\overrightarrow{O_{1} F}$ and $\overrightarrow{\mathrm{OH}}$ are

$$
\begin{aligned}
& \mathbf{n}_{\mathrm{O}_{1} F}=\left(\cos \gamma \sin \phi_{c}, \sin \gamma \sin \phi_{c}, \cos \phi_{c}\right), \\
& \mathbf{n}_{\mathrm{OH}}=\left(\cos \alpha_{0} \sin \beta_{0}, \sin \alpha_{0} \sin \beta_{0}, \cos \beta_{0}\right),
\end{aligned}
$$

where $\gamma=\omega t+\gamma_{0}, \omega=2 \pi f_{m}$ is the rotating angular frequency and $\gamma_{0}$ is the initial phase, both unknown, and $\alpha_{0}$ as well as $\beta_{0}$ are known for parameter estimation in Section 5.

Then, we can easily have

$$
\begin{aligned}
\cos \eta_{\theta} & =\mathbf{n}_{\mathrm{O}_{1} F} \cdot \mathbf{n}_{\mathrm{O}_{1} P_{t}} \approx \mathbf{n}_{\mathrm{O}_{1} F} \cdot \mathbf{n}_{\mathrm{OP}} \approx \mathbf{n}_{\mathrm{O}_{1} F} \cdot \mathbf{n}_{\mathrm{OH}} \\
& =\sin \phi_{c} \sin \beta_{0} \cos \left(\gamma-\alpha_{0}\right)+\cos \phi_{c} \cos \beta_{0},
\end{aligned}
$$

where the approximation is because $O_{1} P_{t}$ is approximately parallel to $O P_{t}$ and even to $\mathrm{OH}$ when the extent of $\theta$ is not large.

We can also easily have

$$
\left|P_{t} O_{1}\right|=\sqrt{\left(V_{a} t-Y_{c}+y\right)^{2}+\left(X_{c}+x\right)^{2}} .
$$

From (3) or (10), we have

$$
\overrightarrow{O_{1} S} \cdot \mathbf{n}_{P_{t} O_{1}}=\frac{P \sin ^{2} \eta_{\theta}}{2 \cos \eta_{\theta}} .
$$

Then using the far-field plane wave assumption and (16) as well as (17), we can obtain the range from $P_{t}$ to $S$ at slow time $t$

$$
\begin{aligned}
R(t) & \stackrel{\text { def }}{=} R_{\theta}=\left|P_{t} O_{1}\right|+\overrightarrow{O_{1} S} \cdot \mathbf{n}_{P_{t} O_{1}} \\
& =\sqrt{\left(V_{a} t-Y_{c}+y\right)^{2}+\left(X_{c}+x\right)^{2}}+\frac{P \sin ^{2} \eta_{\theta}}{2 \cos \eta_{\theta}} \\
& \approx \sqrt{\left(V_{a} t-Y_{c}\right)^{2}+X_{c}^{2}}+x \cos \theta+y \sin \theta+\frac{P \sin ^{2} \eta_{\theta}}{2 \cos \eta_{\theta}}
\end{aligned}
$$

where $\eta_{\theta}$ can be calculated by (15).

After range compression and discarding insignificant factors representing the beam pattern and window functions, SAR returns can be written in the frequency slow time domain as

$$
G(K, \theta)=s(\theta) \exp [-j K R(t)]
$$

When processing spotlight SAR data with algorithms such as the polar format algorithm (PFA), one usually compensates the returns with the scenario center's phase, that is, to multiply (19) with $\exp \left(j K \sqrt{\left(V_{a} t-Y_{c}\right)^{2}+X_{c}^{2}}\right)$. Hence, we obtain the resulting returns

$$
\begin{aligned}
& g(K, \theta)= s(\theta) \exp \left[-j K\left(x \cos \theta+y \sin \theta+\frac{P \sin ^{2} \eta_{\theta}}{2 \cos \eta_{\theta}}\right)\right] \\
&= s(\theta) \exp [-j K(x \cos \theta+y \sin \theta \\
&\left.\left.+\frac{P}{2}\left(\frac{1}{\cos \eta_{\theta}}-\cos \eta_{\theta}\right)\right)\right] \\
&= \sum_{i=0}^{I} b(i) \lambda_{i}(\theta) \quad \\
& \times \exp [-j K(x \cos \theta+y \sin \theta \\
&\left.\left.\quad+\frac{P}{2}\left(\frac{1}{\cos \eta_{\theta}}-\cos \eta_{\theta}\right)\right)\right]
\end{aligned}
$$

which is named the hybrid scattering/motion model, or migratory/micromotion scattering center (MMSC) model, 
where $\lambda_{i}(\theta)$ are orthogonal basis functions. It is of the same form as (10) derived based on a turntable, but herein it is derived from the SAR geometry and its $\eta_{\theta}$ is determined by (15). This model has incorporated motion parameters into the scattering center model, providing the rationale for joint estimation of scattering-motion parameters. It will be seen in next section that the model provides a very good representation of a PRA. By the way, the $2 \mathrm{D}$ inverse FT of (20), interpolation probably needed, will produce an SAR complex image.

Assume that $\theta$ is minor and near $0^{\circ}, \theta_{c}=0^{\circ}$, and $\eta_{\theta}$ is around $0^{\circ}$ or $180^{\circ}$. Then, we have

$$
\frac{1}{\cos \eta_{\theta}} \approx \begin{cases}2-\cos \eta_{\theta}, & \text { when } \eta_{\theta} \text { is around } 0^{\circ}, \\ -2-\cos \eta_{\theta}, & \text { when } \eta_{\theta} \text { is around } 180^{\circ} .\end{cases}
$$

Applying (14), (15), and (21) to (20) leads to

$$
\begin{aligned}
& g(K, \theta) \\
& \approx s(\theta) \exp \left[-j K\left(x \cos \theta+y \sin \theta-P \cos \eta_{\theta}\right.\right. \\
& \left.\left.\quad+P \operatorname{sign}\left(\cos \left(\eta_{\theta}\right)\right)\right)\right] \\
& \approx C \cdot s(\theta) \quad \times \exp [-j K \\
& \left.\quad \times\left(\frac{V_{a} y t}{X_{c}}-P \sin \phi_{c} \sin \beta_{0} \cos \left(\omega t+\gamma_{0}-\alpha_{0}\right)\right)\right],
\end{aligned}
$$

where $C$ is an insignificant unknown constant factor. The instantaneous Doppler, or micro-Doppler, can be obtained by taking the derivative of the phase

$$
f_{d} \approx-\frac{2 \omega P \sin \phi_{c} \sin \beta_{0}}{\lambda} \sin \left(\omega t+\gamma_{0}-\alpha_{0}\right)-\frac{2 V_{a} y}{\lambda X_{c}} .
$$

Both (22) and (23) indicate that the RPRA's azimuth return is an SFM signal, but in effect, it can be approximated by an LFM signal due to broadside flash.

\section{Radar Characteristics of a PRA and Validation of the MMSC Model}

The analysis proceeds with radar characteristics of a PRA, both stationary and rotating, in terms of RCS, HRRPs, TFD, and SAR images. The results from GO, PO, and GRECO are all based on the following geometry and parameters.

As shown in Figure 3, the origin is now set at its geometrical center $\mathrm{O}_{2}$ according to our GRECO software's definition. $\mathrm{O}_{1} \mathrm{O}_{2}=\mathrm{O}_{2} \mathrm{O}_{3}=0.25 \mathrm{~m}$. The PRA's depth $\mathrm{O}_{1} \mathrm{O}_{3}=0.5 \mathrm{~m}$, $P=9 \mathrm{~m}$, and $D=6 \mathrm{~m}$. The frequencies are from $9.8 \mathrm{GHz}$ to $10.2 \mathrm{GHz}$ stepped by $0.01 \mathrm{GHz}$ with central frequency $f_{c}=$ $10 \mathrm{GHz}$ (therefore the bandwidth is $400 \mathrm{MHz}$ and the range resolution is $0.375 \mathrm{~m}$ ), and the aspect angles are from $-180^{\circ}$ to $180^{\circ}$ stepped by $0.1^{\circ}$. Then, we use GRECO to calculate the complex RCS of a PRA on a turntable (equivalent to radar's circling a stationary PRA, at an angular frequency $\omega=\theta^{\prime} / t$ ). We now analyze the calculated results.

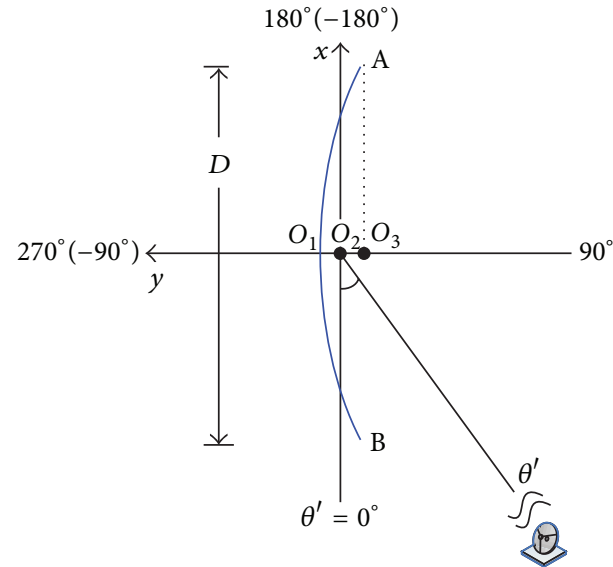

FIgURE 3: PRA geometry for GO, PO and GRECO.

\subsection{Stationary PRA}

4.1.1. RCS Characteristics. As shown by Figures 4(a) and 4(b), the PRA's RCS exhibits strong dependency on aspect angle $\theta^{\prime}$. Its scattering is intense (about $250 \mathrm{~m}^{2}$ or $24 \mathrm{~dB} \cdot \mathrm{m}^{2}$, far larger than the PRA's aperture area $\pi(D / 2)^{2} \approx 28 \mathrm{~m}^{2}$ ) when the LOS deviates from its axis of symmetry by no more than about $18^{\circ}$. Otherwise its RCS abruptly declines to zero. This phenomenon is generally called broadside flash [16]. From $(4),|\eta| \leq \arctan [6 /(2 \times 9)] \approx 18.4^{\circ}$, which agrees with GRECO results (solid line) and means that GO has correctly predicted the aspect supporting interval. Figure 4(c) shows that GO can also give a coarse prediction for the RCS $-\theta^{\prime}$ function by frequency-free (1). For more accurate description, $s(\theta)$ is therefore used in (10).

From Figure 4(b), we can also find that the RCS $-\theta^{\prime}$ curves are almost identical for different frequencies, except when $\theta^{\prime}= \pm 90^{\circ}$, indicating weak frequency dependency of RCS. This is confirmed by Figure 5(a), which shows the RCSfrequency functions are valued between 22 and 26 in $\mathrm{dBm}^{2}$, a small range, regardless of aspects (except $\theta^{\prime}= \pm 90^{\circ}$ ). Therefore, our MMSC model, though not considering RCS's dependency on frequency, that is, $s(\theta)$ does not contain $f$ in (20), is judged reasonable from this viewpoint. However, when the LOS is perpendicular to the PRA's bottom, RCS takes on obvious oscillation for PO and GRECO (Figure 5(b)). The reason may be stated in terms of multiple scatterers; that is, there may be additional scattering centers besides the specular point predicted by GO at this aspect.

4.1.2. HRRP Characteristics. Figure 6 depicts the HRRP sequence of the PRA, derived from GRECO data. Since HRRPs at some aspects are too weak to display in Figure 6(a), we modify their amplitudes for every aspect into a normalized value, as shown in Figure 6(b). We will analyze its HRRP characteristics from the following points.

(1) Overall, strong HRRPs occur periodically when $\theta^{\prime} \epsilon$ $\left[-108^{\circ},-72^{\circ}\right] \cup\left[72^{\circ}, 108^{\circ}\right]$, which is consistent with our analysis of RCS. The HPPRs' intensity is almost identical for front and back illumination. 


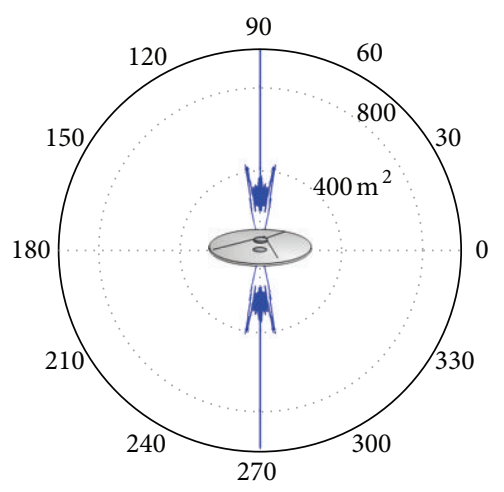

(a)
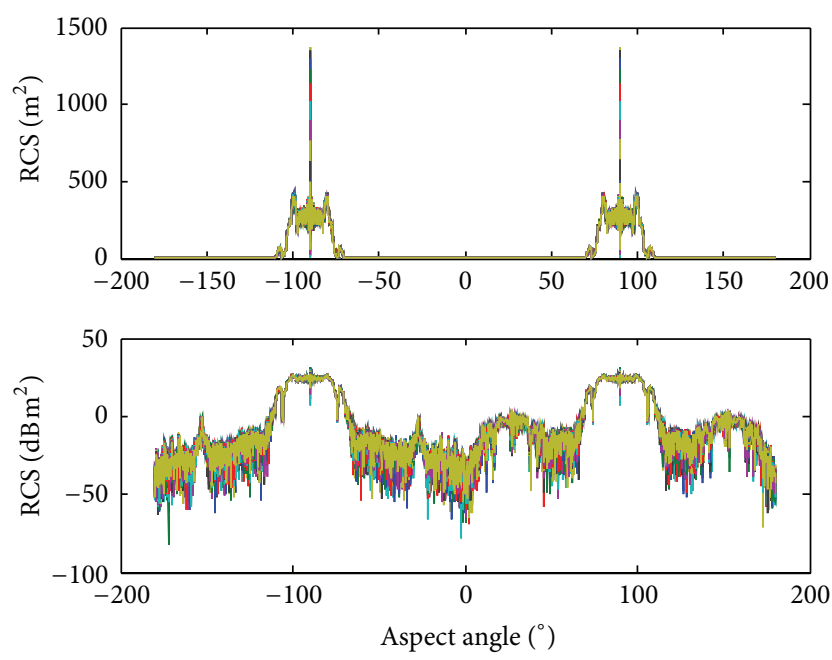

(b)

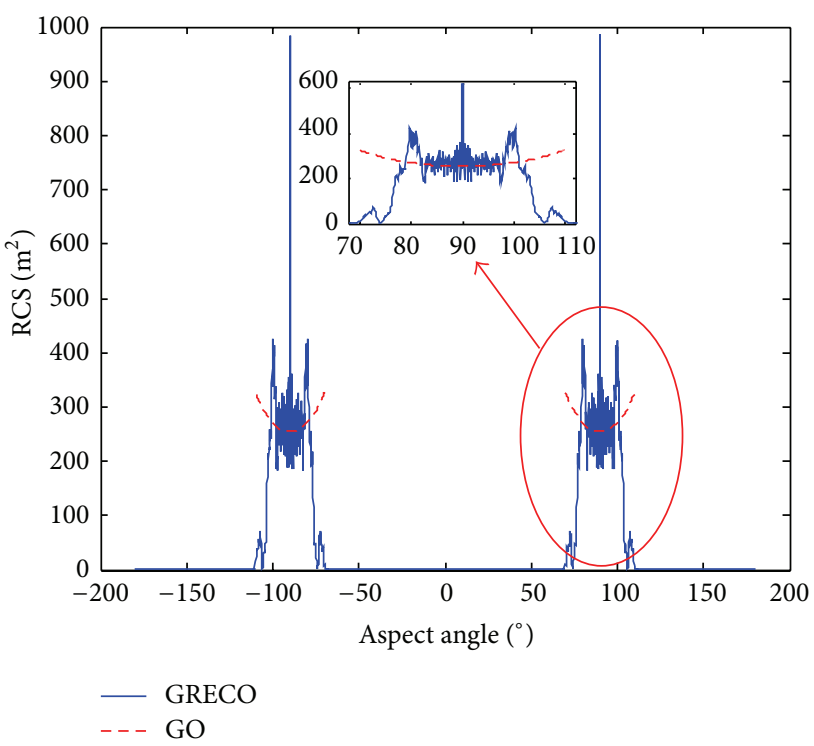

(c)

FIGURE 4: PRA RCS's aspect dependency. (a) GRECO, at carrier frequency $10 \mathrm{GHz}$; (b) GRECO, at different frequencies; (c) GRECO and $\mathrm{GO}$, at carrier frequency $10 \mathrm{GHz}$.

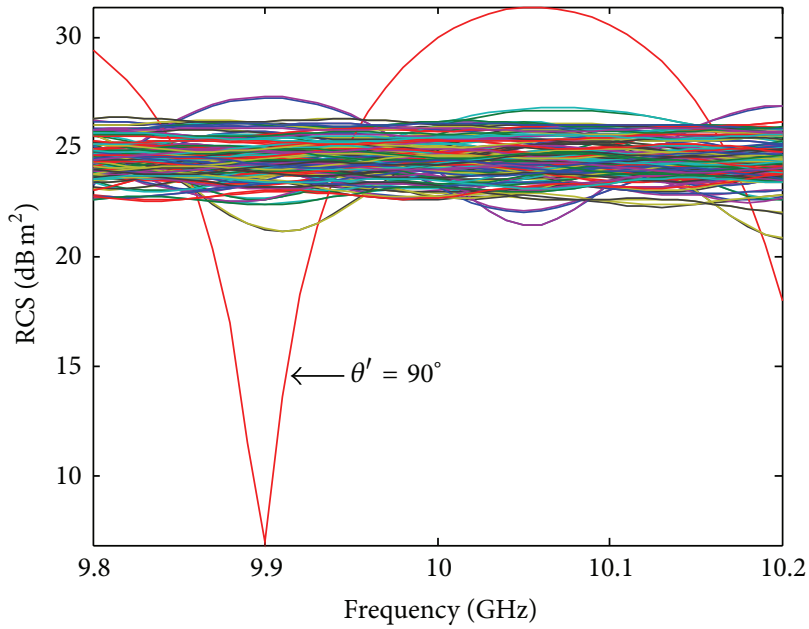

(a)

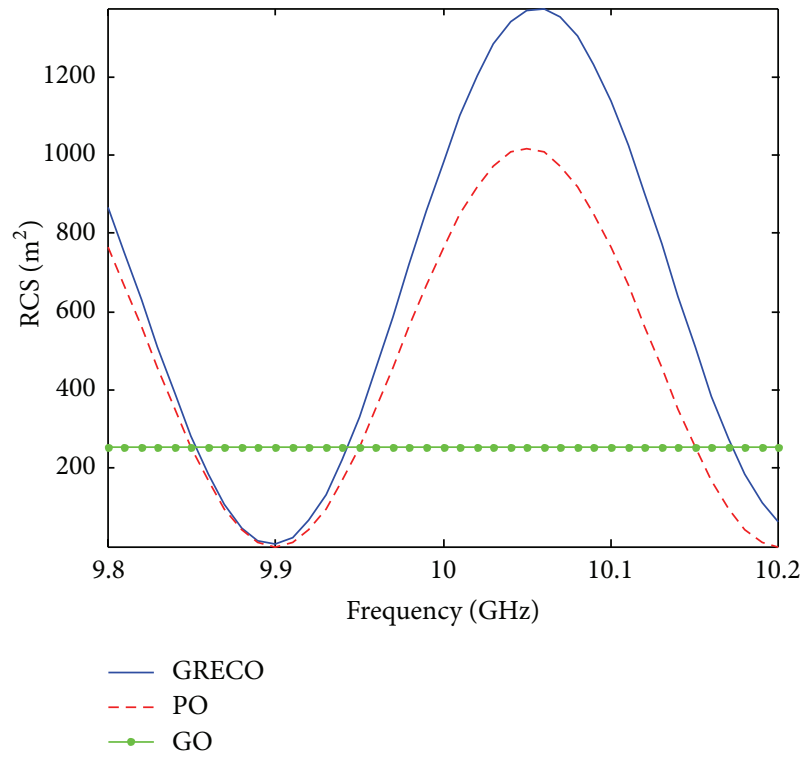

(b)

FIGURE 5: RCS's frequency dependency. (a) GRECO, at different aspects; (b) GRECO, PO, and GO, $\theta^{\prime}=90^{\circ}$.

(2) When illuminated broadside $\left(\theta^{\prime}= \pm 90^{\circ}\right)$, the PRA has two scattering centers at $\pm 0.25 \mathrm{~m}$ with the same amplitude (two peaks in Figure 6(a); only for this aspect, the bandwidth is $2 \mathrm{GHz}$ as opposed to $400 \mathrm{MHz}$ to resolve them). This can be interpreted by (9) given by PO, the second square-bracketed term of which clearly shows that the positions of the two scattering centers are $\pm D^{2} /(16 P)= \pm 0.25 \mathrm{~m}$. And the conclusion also confirms our conjecture on the reason of RCS oscillation in Figure 5(b). In effect, the two centers correspond to the PRA's bottom center and edge.

(3) When $\theta^{\prime} \in\left[-108^{\circ},-72^{\circ}\right] \cup\left[72^{\circ}, 108^{\circ}\right]$ and $\theta^{\prime} \neq \pm 90^{\circ}$, the PRA assumes a single scattering center. When the PRA's front side is illuminated, the center moves 


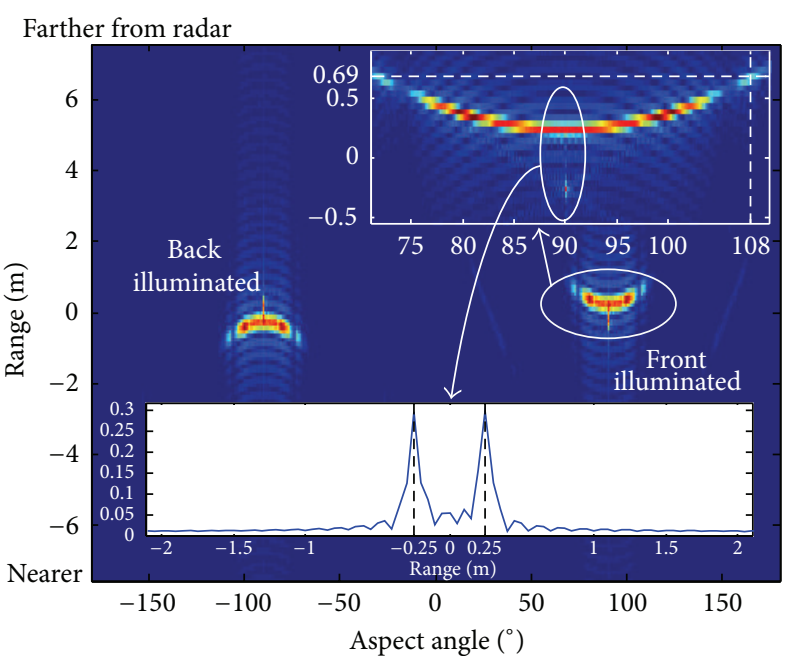

(a)

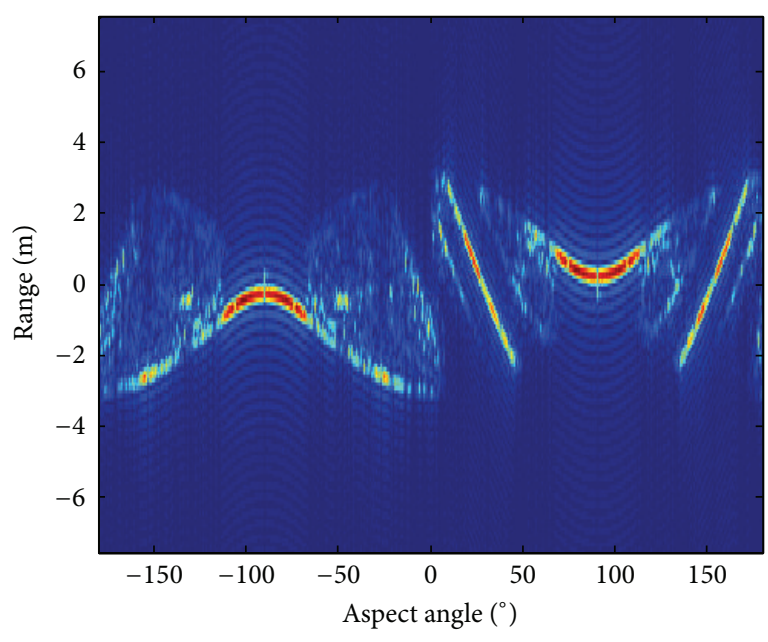

(b)

FIGURE 6: HRRP sequences of the PRA with (a) original and (b) normalized amplitude. GRECO data are used.

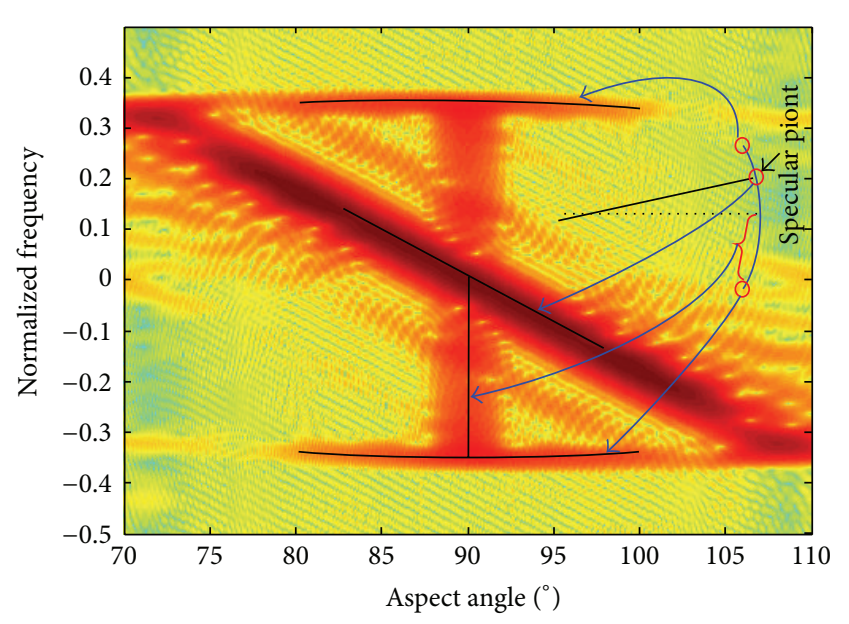

(a)

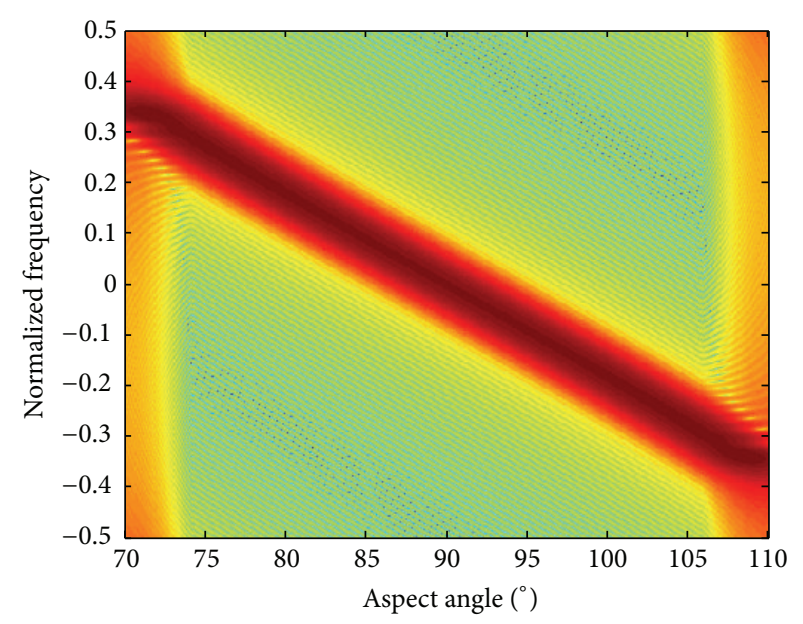

(b)

FIGURE 7: TFD of the PRA with data from (a) GRECO and (b) our PRA scattering center model.

further with deviation of the LOS from the PRA's symmetry axis. The case is just the opposite when the back side is illuminated. This conclusion also agrees with our model's prediction (see (10) and (11)). More specifically, in Figure 3, when $\theta^{\prime}=108^{\circ}$, the scattering center should move to $\sin \theta^{\prime} / 4+P \cos ^{2} \theta^{\prime} /\left(2 \sin \theta^{\prime}\right) \approx$ $0.69 \mathrm{~m}$, which is in good agreement with the reality in the top-right corner of Figure 6(a). Beyond this range, the center will move outside the PRA and therefore disappear.

(4) When $\theta^{\prime} \notin\left[-108^{\circ},-72^{\circ}\right] \cup\left[72^{\circ}, 108^{\circ}\right]$, the HRRPs are too weak and we have to resort to Figure 6(b). Clearly, for these aspects, the scattering is rather complicated due to disappearing of the specular point, and more scattering centers are present beyond the prediction of GO and our models. Fortunately, these HRRPs are so weak that they can be safely ignored.
4.1.3. TFD Characteristics. We also plot the TFD according to the PRA's complex RCS at the central frequency, only for aspects at which the PRA's RCS is strong. The PRA is placed on a "rotatable" turntable, and thus the aspect angle has the sense of slow time. Figure 7(a) shows the TFD based on GRECO-derived data. it is clear that the PRA contains three main scattering centers. One is the specular point, related to the slant line in Figure 7(a). The slant line agrees well with (12) and its graphical presentation in Figure 7(b). Two is at the PRA's edge, that is, points $A$ and $B$ in Figure 3, related to the top and bottom curves in Figure 7(a). Each curve is effectively a small fraction of a sine wave. We now try to explain the vertical line in the middle of Figure 7(a). When $\theta^{\prime}=90^{\circ}$, different parts of the PRA have widerange relative velocities, from 0 corresponding $O_{1}$ to the maximum and minimum corresponding to point $A$ and $B$ in Figure 3. Therefore the instantaneous Doppler at $\theta^{\prime}=$ 


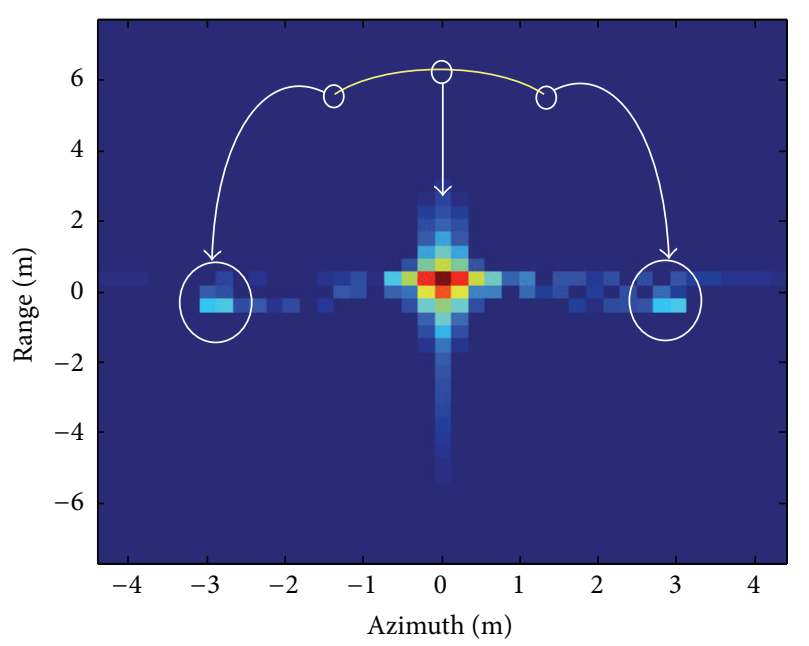

(a)

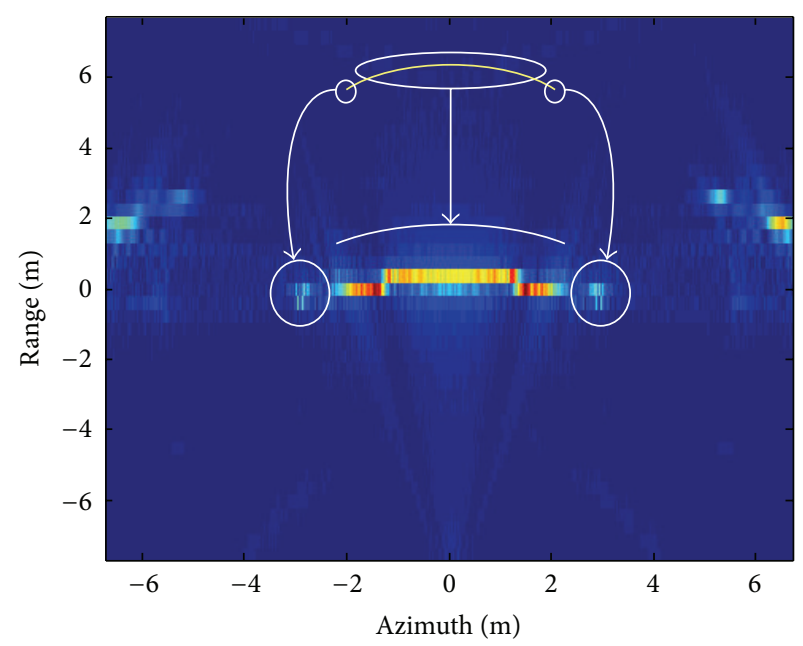

(b)

Figure 8: 2D images of the PRA in a turntable, by CBP, using GRECO data. (a) $\theta^{\prime} \in\left[88^{\circ}, 92^{\circ}\right]$; (b) $\theta^{\prime} \in\left[0^{\circ}, 180^{\circ}\right]$.

TABLE 1: SAR and RPRA's parameters.

\begin{tabular}{lccc}
\hline Bandwidth & $400 \mathrm{MHz}$ & Reference range $X_{c}$ & $10000 \mathrm{~m}$ \\
Central frequency $f_{c}$ & $10 \mathrm{GHz}$ & PRA's focus parameter $P$ & $9 \mathrm{~m}$ \\
Slant angle $\theta_{c}$ & 0 & Diameter of the PRA's aperture $D$ & $6 \mathrm{~m}$ \\
CPI & $8.7 \mathrm{~s}$ & PRA rotating frequency $f_{m}$ & $0.1 \sim 0.3 \mathrm{~Hz}$ \\
Airplane velocity $V_{a}$ & $200 \mathrm{~m} / \mathrm{s}$ & $\phi_{c}$ & $\pi / 4$ \\
Angular extent & $10^{\circ}$ & $\gamma_{0}$ & $\pi$ \\
\hline
\end{tabular}

$90^{\circ}$ contains all the components throughout between the maximal, and minimal Doppler, leading to a vertical line in Figure 7(a). In addition, it is clear that, though there are several components in Figure 7(a), the slant line has the strongest intensity. Thereby it is reasonable for our scattering center models to contain only the specular scattering component.

4.1.4. 2D Image Characteristics. The $2 \mathrm{D}$ images of the PRA are formulated by the convolution back-projection (CBP) algorithm, displayed in Figure 8 for narrow and wide angles, respectively. From Figure 8(a), three scattering centers are present, with the middle bright one at the PRA's bottom center and the other darker two at its edge. This is in accordance with Figure 7(a). However, in the wide-angle case (Figure 8(b)), the PRA exhibits a bowl-shaped signature, and the bowl's size is equal to the true size of the PRA. The two points corresponding to the PRA's edge are still visible at the bowl's edge. The bowl shape is due to the migration of the specular point within such a wide angle. From Figure 6 it is well known at different aspects, that the specular point is located at different positions, and therefore they cannot be coherently integrated into a single strong point in the resulting image. Instead, the energy is spread over the bowl shape. Moreover, there is an other weak energy distributed at the left and right edges of Figure 8(b). This energy results from other types of scattering from the PRA, for example, creeping wave. Again, the PRA, when its back side faces radar, will assume similar signatures to Figure 8, but the bowl's direction is the opposite. To summarize, 2D images provide a very good representation of PRA's structure, and the specular scattering contributes to PRA's dominant energy.

4.2. Rotating PRA. When a PRA is rotating in the SAR geometry, its radar characteristics will considerably vary due to the rotation. In what follows, we proceed with the analysis of an RPRA based on the MMSC model since the real SAR data of an RPRA are unavailable for the moment. Simulation parameters are shown in Table 1.

4.2.1. RCS, HRRP, and TFD Characteristics. Suppose $f_{m}=$ $0.3 \mathrm{~Hz}$, which is also a typical value for real RPRAs. In Figure 9(a), as is expected, the RPRA's RCS sequence takes on periodicity due to the rotation and the period is about $3.3 \mathrm{~s}$, in good agreement with the true value $1 / f_{m}$. Its TFD also shows the periodicity (Figure 9(b)). In any segment, the slantlineshaped TFD represents an LFM signal, or a fraction of a SFM signal, as expected by (23). In effect, a sine wave is impossible to be displayed in the TF image owing to broadside flash. Note also that the slant lines may be blurry due to short time and the TF-resolution limit. This kind of periodicity is also characteristic of the HRRP sequence in Figure 9(c). However, a curve similar to the left one in Figure 6(a) is not present. This is because that the back of the PRA has no chance to be illuminated by SAR for the parameters in Table 1. 


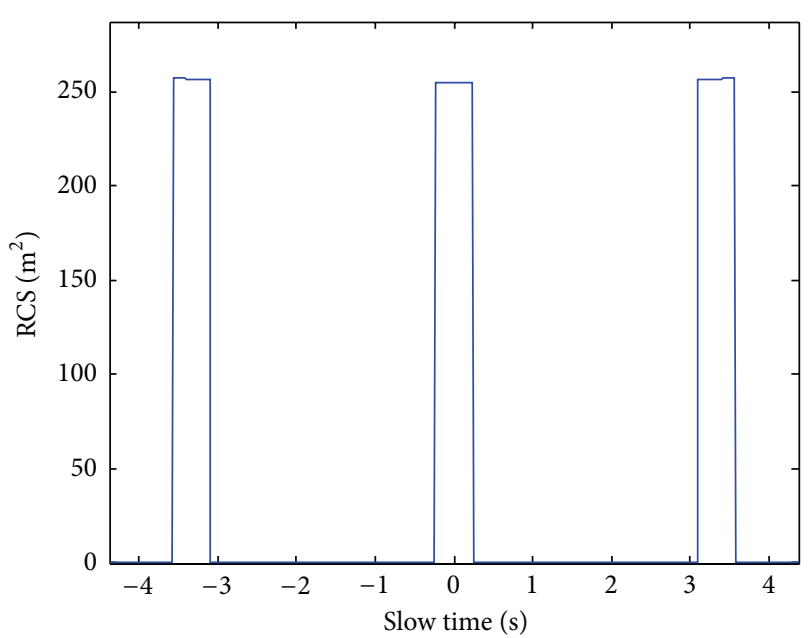

(a)

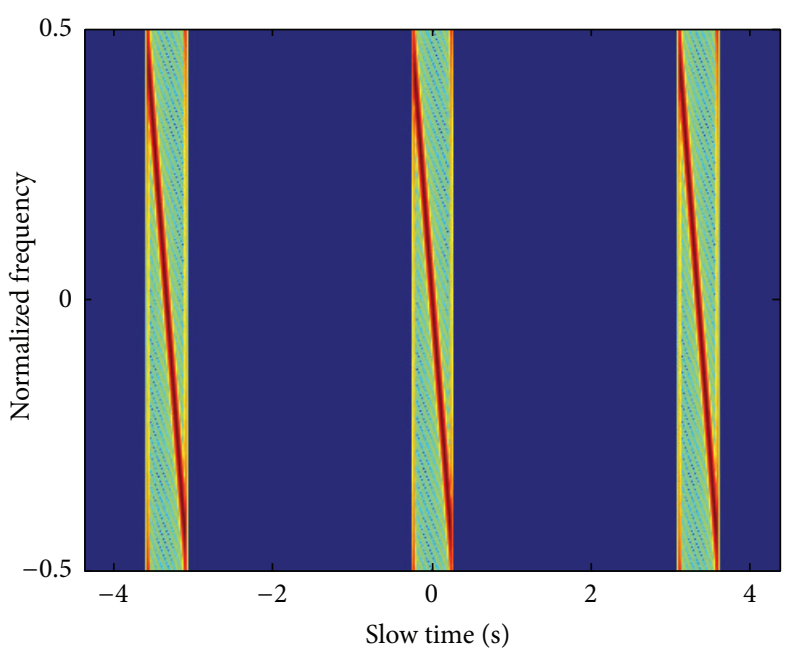

(b)

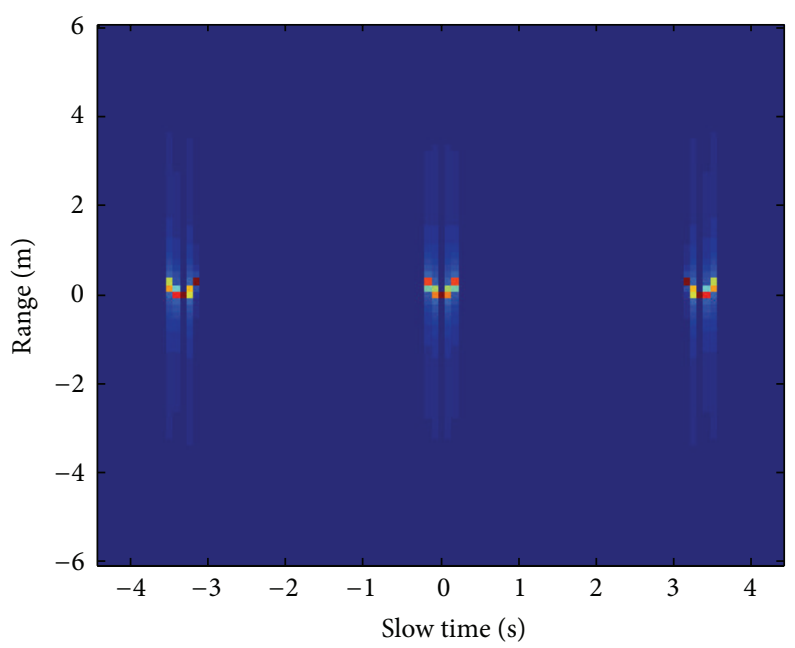

(c)

FIGURE 9: RPRA's characteristics. (a) RCS sequence; (b) TFD; (c) HRRP sequence.

4.2.2. SAR Image Characteristics. The presence of rotation changes the Doppler history of the PRA's returns, thus bringing out new signatures distinct from of the stationary PRA. When the PRA rotates at a small frequency, it takes on blurred lines in the SAR image (Figure 10(a)). When it rotates more rapidly, the image takes on a bowknot shape with several intersectant lines (Figure 10(b)). The faster the PRA rotates, the more lines there are. When $f_{m}=2 \mathrm{~Hz}$, we cannot distinguish these lines from each other. As a result, the image exhibits another kind of bowknots as shown in Figure 10(c). We also find that the angular extent of the bowknots is approximately equal to that of SAR, that is, $10^{\circ}$, in Figures 10(b) and 10(c), after the units of the two axes are equalized. These interesting signatures can provide new features for target recognition, notwithstanding that the underlying reason is enigmatic and remains to be explored in the future.

4.2.3. Effective Illumination Condition. In Figure 9(c), we have found that the back of the PRA is not illuminated by SAR. In effect, even if it is illuminated, there is not backscattering strong enough if the incident angle is too large. In such a case, the illumination is not effective. Then, what is the condition of effective illumination? Clearly, $\left|\eta_{\theta}\right| \leq \Delta$ or $\pi-\Delta \leq\left|\eta_{\theta}\right| \leq \pi+\Delta$ needs and only needs to be satisfied where $\Delta$ is half the aspect supporting interval (e.g., $18^{\circ}$ ). Substituting it into (15) and after some simple manipulations, we find that the sufficient and necessary conditions for effective front or back illumination are, respectively,

$$
\begin{gathered}
\left|\beta_{0}-\phi_{c}\right| \leq \Delta \\
\beta_{0}+\phi_{c} \geq \pi-\Delta .
\end{gathered}
$$

If (24) and (25) are combined, we readily obtain a necessary condition of both sides being illuminated

$$
\Delta \geq \frac{\pi}{3} \text {. }
$$

However, in general, $\Delta<\pi / 4$ for PRAs. Therefore, it is impossible for both sides of a PRA to be effectively illuminated. 


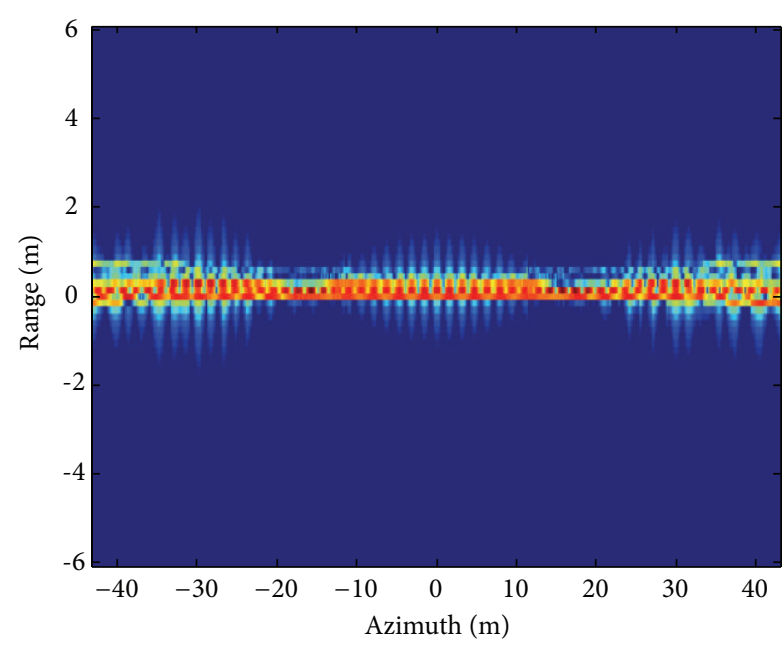

(a)

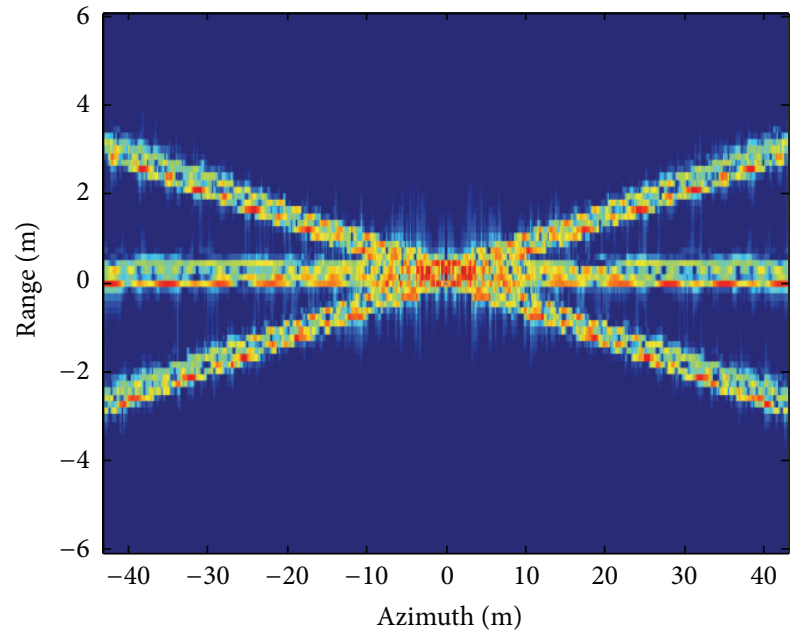

(b)

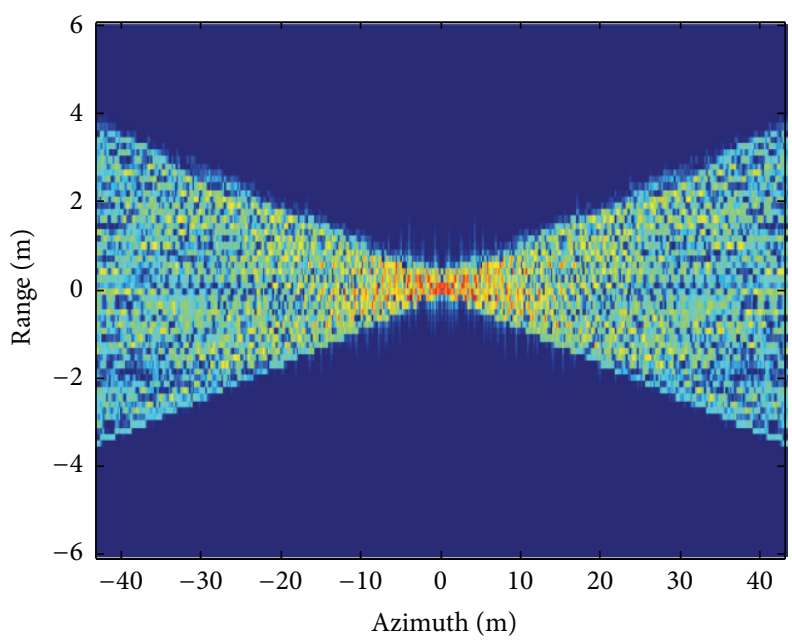

(c)

FIGURE 10: SAR images of an RPRA, formulated by CBP, when (a) $f_{m}=0.2 \mathrm{~Hz}$, (b) $f_{m}=0.3 \mathrm{~Hz}$, and (c) $f_{m}=2 \mathrm{~Hz}$.

The analyses made earlier have fully supported our scattering center models and provided deep insight into PRA's characteristics. These characteristics can be used as feature for SAR image interpretation and target detection or recognition. They can also be used for parameter's coarse estimation as shown in Section 5.

\section{MLE of Parameters in the MMSC Model}

Once the MMSC model is built, the estimation for parameters in this model can be performed from measured data, and there are various ways available, for example, MLE [14], the regularization method [17], and the sparse representation method [15], though they were not intended for our problem herein. Actually, from a unified viewpoint, all of them belong to optimization algorithms for solving inverse problems. In this paper, we develop an MLE method for the MMSC model, which is equivalent to the nonlinear least squares estimation [14]. Note that only one PRA target is assumed for simplicity, but it can be generalized for multiple targets by introducing RELAX or CLEAN techniques, thereby circumventing the estimation of the model order.
5.1. Maximal Likelihood Estimator. In $(20), s(\theta)$ has been parameterized in basis functions. For notational expediency, (20) can be further vectorized as

$$
\mathbf{g}=\boldsymbol{\Phi}(\vartheta) \mathbf{b}+\mathbf{v}
$$

where $\mathbf{g}$ is the measured echo vector, $\mathbf{v}$ is the noise vector, $\vartheta \stackrel{\text { def }}{=}\left(x, y, f_{m}, \gamma_{0}, \phi_{c}, P\right)$ is unknown, $\mathbf{b} \stackrel{\text { def }}{=}[b(0), \ldots, b(I)]^{T}$ is unknown, and

$$
\begin{aligned}
& e_{K \theta} \stackrel{\text { def }}{=} \exp \left[-j K\left(x \cos \theta+y \sin \theta+\frac{P \sin ^{2} \eta_{\theta}}{2 \cos \eta_{\theta}}\right)\right], \\
& \boldsymbol{\Phi} \stackrel{\operatorname{def}}{=}\left[\begin{array}{ccc}
e_{K_{1}} \theta_{1} & 0 \\
\ddots & \ddots \\
0 & e_{K_{1} \theta_{M}} \\
& \ldots & \\
e_{K_{N}} \theta_{1} & 0 \\
& \ddots \\
0 & e_{K_{N} \theta_{M}}
\end{array}\right]\left[\begin{array}{ccc}
\lambda_{0}\left(\theta_{1}\right) & \ldots & \lambda_{O}\left(\theta_{1}\right) \\
\vdots & \vdots & \vdots \\
\lambda_{0}\left(\theta_{M}\right) & \ldots & \lambda_{O}\left(\theta_{M}\right)
\end{array}\right],
\end{aligned}
$$




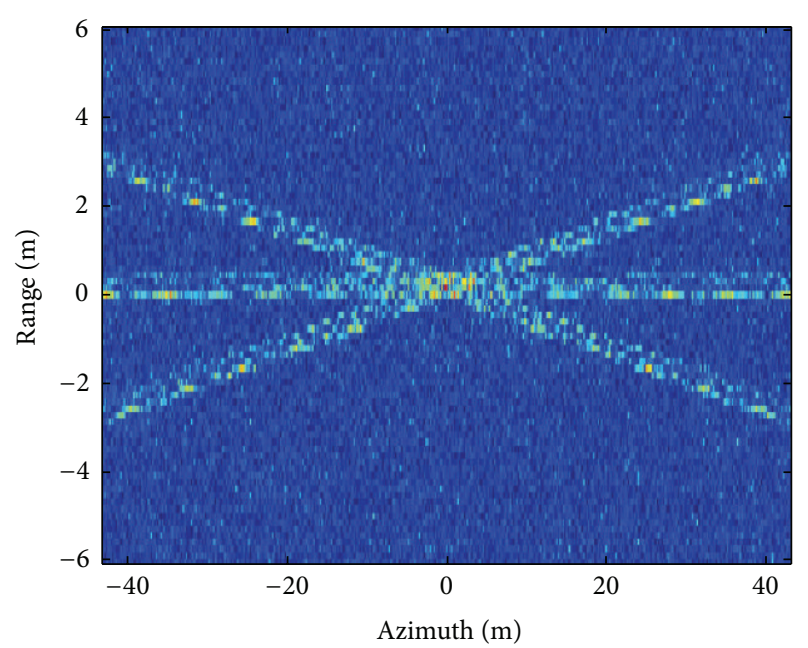

FIgURE 11: PRA's image under $\mathrm{SNR}=-3 \mathrm{~dB}$, by CBP.

TABLE 2: Final estimated results.

\begin{tabular}{lccc}
\hline & $x(\mathrm{~m})$ & $y(\mathrm{~m})$ & $f_{m}(\mathrm{~Hz})$ \\
\hline True value & 0 & 0 & 0.3 \\
Coarse estimate & 0.12 & -0.10 & 0.32 \\
Estimate & 0.0013 & 0.0108 & 0.2995 \\
\hline & $\gamma_{0}(\mathrm{rad})$ & $\phi_{c}(\mathrm{rad})$ & $P(\mathrm{~m})$ \\
\hline True value & $\pi / 2$ & $\pi / 4$ & 9 \\
Coarse estimate & $0: \pi / 4: 7 \pi / 4$ & 0.68 & 10 \\
Estimate & 1.5715 & 0.7841 & 9.0179 \\
\hline
\end{tabular}

where the derivation of $\Phi$ is not difficult but tedious, therefore not given here.

We try to solve for 9 and $\mathbf{b}$ from (27). Clearly, this is an inverse problem. Using MLE, we at first obtain $\vartheta$ 's estimate

$$
\widehat{\vartheta}=\underset{\vartheta}{\arg \min }\left\|\mathbf{g}-\boldsymbol{\Phi}\left(\boldsymbol{\Phi}^{H} \boldsymbol{\Phi}\right)^{-1} \boldsymbol{\Phi}^{H} \mathbf{g}\right\|_{2}^{2} .
$$

Afterwards, by substituting $\widehat{\vartheta}$ into $\Phi$, we have the leastsquares estimate of $\mathbf{b}$

$$
\widehat{\mathbf{b}}=\left(\boldsymbol{\Phi}^{H} \boldsymbol{\Phi}\right)^{-1} \boldsymbol{\Phi}^{H} \mathbf{g} .
$$

Finally, by use of $\widehat{\mathbf{b}}$, the reflectivity function can be found as

$$
\widehat{\mathcal{s}}(\theta)=\sum_{i=0}^{I} \widehat{b}(i) \lambda_{i}(\theta) .
$$

5.2. Determination of the Initial Value of $\vartheta$. Equation (29) is an optimization function, and it is easy to cause local minima. One may either try to find efficient global optimization algorithms, which have not been found to date, or to determine initial values with higher precision. The latter line of thought entails a thorough understanding of the target characteristics, which, fortunately, has been analyzed in detail in Section 4. Therefore, we give the coarse estimation method for $\vartheta$.

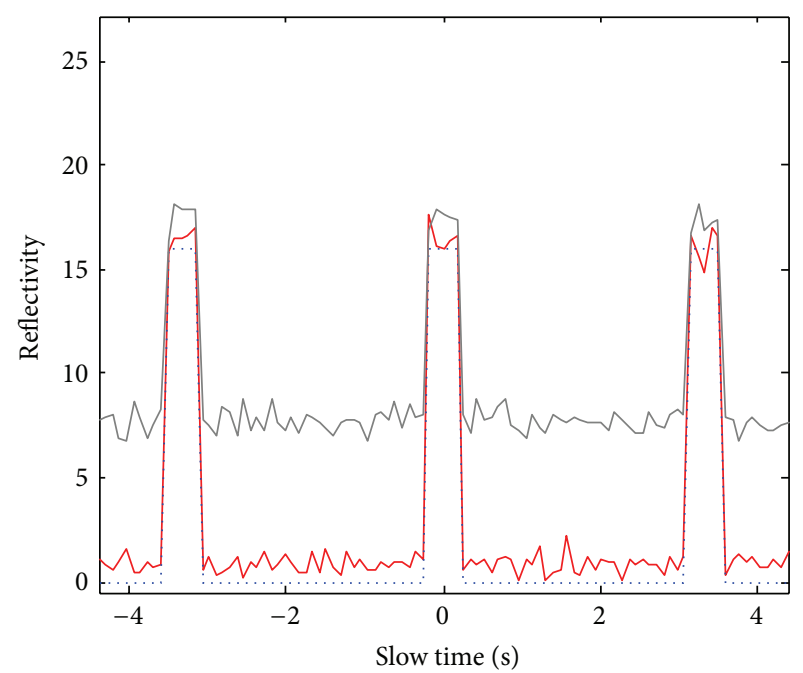

_... Our method
$\ldots$ Ture
$\ldots$ Conventional method

FIGURE 12: Recovered reflectivity with respect to slow time.

$(x, y)$ : From Figure 10, the center of an RPRA's blurred image indicates the position of the scattering center. So, $(x, y)$ can be estimated, respectively, by

$$
\begin{aligned}
& x=\frac{\iint x^{\prime} \cdot I\left(x^{\prime}, y^{\prime}\right) d x^{\prime} d y^{\prime}}{\iint I\left(x^{\prime}, y^{\prime}\right) d x^{\prime} d y^{\prime}} \\
& y=\frac{\iint y^{\prime} \cdot I\left(x^{\prime}, y^{\prime}\right) d x^{\prime} d y^{\prime}}{\iint I\left(x^{\prime}, y^{\prime}\right) d x^{\prime} d y^{\prime}},
\end{aligned}
$$

where $I\left(x^{\prime}, y^{\prime}\right)$ is the gray-level image.

$f_{m}$ : It can be estimated by finding the rotating period from an HRRP sequence (see Figure 10) and then taking its reciprocal.

$\phi_{c}$ and $P$ : We have not found any effective method for estimating them separately. However, from (23), after obtaining $f_{m}$ and $\omega$, we can find $P \sin \phi_{c}$ as a whole by estimating the Doppler slope. $P$ can be set as a mean value from a priori knowledge, at the order of meters. $\phi_{c}$ can then be determined accordingly.

$\gamma_{0}$ : It is hard to be estimated. We just take a set of values of it as the coarse estimates; so, the objective function will be optimized with different initial values, resulting in multiple local minima, the minimum of which is finally taken as the global minimum. We find through simulation that the optimization is not sensitive to the choice of $\gamma_{0}$ 's initial values.

\section{Simulation Results}

In this section, we present some estimation results by the proposed method in Section 5. $f_{m}=0.3 \mathrm{~Hz}, \alpha_{0}=\pi$, $\beta_{0}=\pi / 4$, the signal-to-noise ratio (SNR) is $-3 \mathrm{~dB}$, and other parameters are shown in Table 1. The Dirac function is chosen as the basis function. The optimization algorithm we use is 


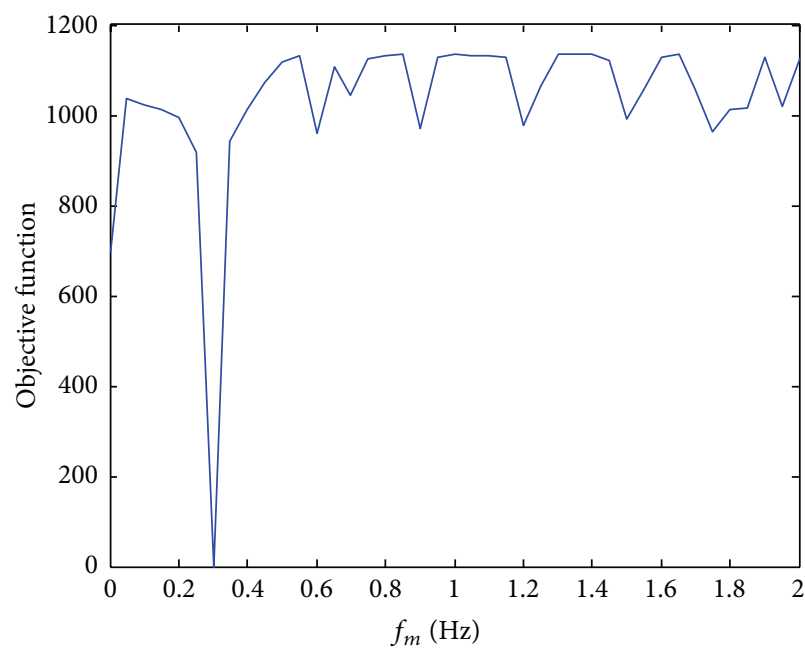

(a)

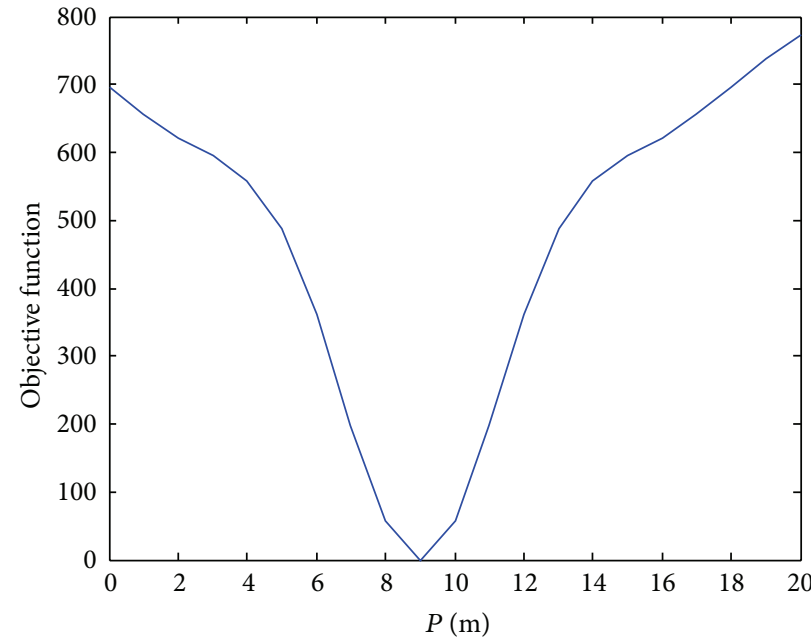

(b)

FIGURE 13: Objective function slices along (a) the rotating frequency and (b) the focus parameter.

sequential quadratic programming (SQP), which has proven successful in solving our problem.

The RPRA's SAR image is shown in Figure 11, which is similar to Figure 10(b) except for being contaminated by noise. The coarse estimates for initial values are shown in Table 2. Using the estimator proposed, we finally obtain the estimates of the PRA's parameters, which, obviously, agree well with their true values. To our best knowledge, there is not any published work on extracting the overall information of an RPRA in SAR and so, overall comparison cannot be performed. Once the six parameters are obtained, the coefficients of the basis functions can be found and therefore the reflectivity function $\widehat{s}(\theta)$ results (Figure 12 ). Note that $\theta$ is proportional to slow time by (14). Figure 12 indicates that the reflectivity is successfully recovered, and the periodicity implies the broadside flash signature which can be favorably used in target recognition. Also, the square of the reflectivity is accordant with the RCS sequence in Figure 9(a). Although the conventional method also gives estimates the reflectivity by evaluating the mean of the returns' amplitude sequence, it has poorer agreement with the reality, and more problematically, this method needs finding range cells containing micromotion targets at first, which is in itself a tough job but is now circumvented by our approach through building the MMSC model in the phase domain.

Again, we can see through the slices of the objective function shown by Figure 13 that improper initial values of $f_{m}$ will lead to local minima, but the coarsely estimating precision for it is able to guarantee the convexity of the objective function within a necessary interval. it is similar to other parameters except $P$ which tolerates larger error of initial estimates (Figure 13(b)).

\section{Conclusion}

As a special type of targets in SAR, RPRAs have a few interesting characteristics which can be exploited for understanding SAR images. In this paper, we have at first obtained the closed-form and numeric solutions for PRA's backscattering, via GO, PO, and GRECO, respectively. From its GO solution, a new scattering center model has been proposed for characterizing the migration of the specular point on the PRA, and then the rotating parameters have been incorporated into the model to obtain a hybrid model or MMSC model in SAR geometry. Using the models and the scattering data calculated, we have in detailed investigated the radar characteristics of a PRA and RPRA alike. Their RCS, HRRP sequences, and TFD all show that a PRA exhibits broadside flash as well as periodicity and contains a single migratory scattering center for limited aspects, whose energy is dominant. The bowl-shaped image of a stationary PRA reflects its structure perfectly, while the SAR image of an RPRA assumes distinct signatures. All these characteristics have confirmed the MMSC model, and they also provide novel information on targets' structures and motions. Then, using this information for coarse estimation of PRA's parameters, we have developed a maximal likelihood estimator to solve the MMSC model. Simulation results at $-3 \mathrm{~dB}$ of SNR demonstrate the estimator's performance. The parameters estimated, for example, the rotating frequency and focus parameter, can faithfully record the information on PRA's motion and size and therefore can be used advantageously as features for target recognition. However, we have concentrated only on a PRA in a homogeneous scenario which can be characterized by white Gaussian noise and lacked consideration of clutters stronger and more complex. Additionally, selection of basis functions has not been discussed, either. Notwithstanding its limitation, this study provides a basis for interpretation of SAR images containing PRAs, and the idea of hybrid modeling is also instrumental for other fields such as SAR/GMTI.

\section{Acknowledgment}

The authors acknowledge the support from Provincial Science Foundation for Distinguished Young Scholars in Hunan 
(no. 11JJ1010) and National Science Fund of China (no. 61171133, no. 61101182, and no. 61025006).

\section{References}

[1] W. J. Miceli and L. A. Gross, "Test results from the AN/APY6 SAR/GMTI surveillance, tracking and targeting radar," in Proceedings of the IEEE Radar Conference, pp. 13-17, Atlanta, Ga, USA, May 2001.

[2] X. Li, B. Deng, Y. Qin, H. Wang, and Y. Li, "The influence of target micromotion on SAR and GMTI," IEEE Transactions on Geoscience and Remote Sensing, vol. 49, no. 7, pp. 2738-2751, 2011.

[3] B. Deng, G. Wu, Y. Qin, H. Wang, and X. Li, "SAR/MMTI: an extension to conventional SAR/GMTI and a combination of SAR and micromotion techniques," in Proceedings of the IET International Radar Conference, Guilin, China, April 2009.

[4] S. D. Silverstein and C. E. Hawkins, "Synthetic aperture radar image signatures of rotating objects," in Proceedings of the Conference Record of the Thirty-Eighth Asilomar Conference on Signals, Systems and Computers, pp. 1663-1667, November 2004.

[5] T. Thayaparan, S. Abrol, and S. Qian, "Micro-doppler analysis of rotating target in SAR,” Tech. Rep. TM-2005, DRDC, Ottawa, Canada, 2005.

[6] T. Thayaparan, K. Suresh, S. Qian, K. Venkataramaniah, S. SivaSankaraSai, and K. S. Sridharan, "Micro-Doppler analysis of a rotating target in synthetic aperture radar," IET Signal Processing, vol. 4, no. 3, pp. 245-255, 2010.

[7] M. Rüegg, E. Meier, and D. Nüesch, "Vibration and rotation in millimeter-wave SAR," IEEE Transactions on Geoscience and Remote Sensing, vol. 45, no. 2, pp. 293-304, 2007.

[8] D. C. Jenn, Radar and Laser Cross Section Engineering, American Institute of Aeronautics and Astronautics, 2005.

[9] J. M. Rius, M. Ferrando, and L. Jofre, "High-frequency RCS of complex radar targets in real-time," IEEE Transactions on Antennas and Propagation, vol. 41, no. 9, pp. 1308-1319, 1993.

[10] M. Hurst and R. Mittra, "Scattering center analysis via prony's method," IEEE Transactions on Antennas and Propagation, vol. 35, no. 8, pp. 986-988, 1987.

[11] L. C. Potter, D. M. Chiang, R. Carriere, and M. J. Gerry, "GTDbased parametric model for radar scattering," IEEE Transactions on Antennas and Propagation, vol. 43, no. 10, pp. 1058-1067, 1995.

[12] L. C. Potter and R. L. Moses, "Attributed scattering centers for SAR ATR," IEEE Transactions on Image Processing, vol. 6, no. 1, pp. 79-91, 1997.

[13] M. J. Gerry, L. C. Potter, I. J. Gupta, and A. D. Van Merwe, "A parametric model for synthetic aperture radar measurements," IEEE Transactions on Antennas and Propagation, vol. 47, no. 7, pp. 1179-1188, 1999.

[14] R. Jonsson, A. Genell, D. Lösaus, and F. Dicander, "Scattering center parameter estimation using a polynomial model for the amplitude aspect dependence," in Proceedings of the Algorithms for Synthetic Aperture Radar Imagery IX, pp. 46-57, Orlando, Fla, USA, April 2002.

[15] K. R. Varshney, M. Çetin, J. W. Fisher, and A. S. Willsky, "Sparse representation in structured dictionaries with application to synthetic aperture radar," IEEE Transactions on Signal Processing, vol. 56, no. 8, pp. 3548-3561, 2008.

[16] R. D. Chaney, A. S. Willsky, and L. M. Novak, "Coherent aspectdependent SAR image formation," in Proceedings of the SPIE
Algorithms for Synthetic Aperture Radar Imagery, vol. 2230, pp. 256-274, 1994.

[17] B. D. Rigling, "Use of nonquadratic regularization in fourier imaging," IEEE Transactions on Aerospace and Electronic Systems, vol. 45, no. 1, pp. 250-265, 2009. 

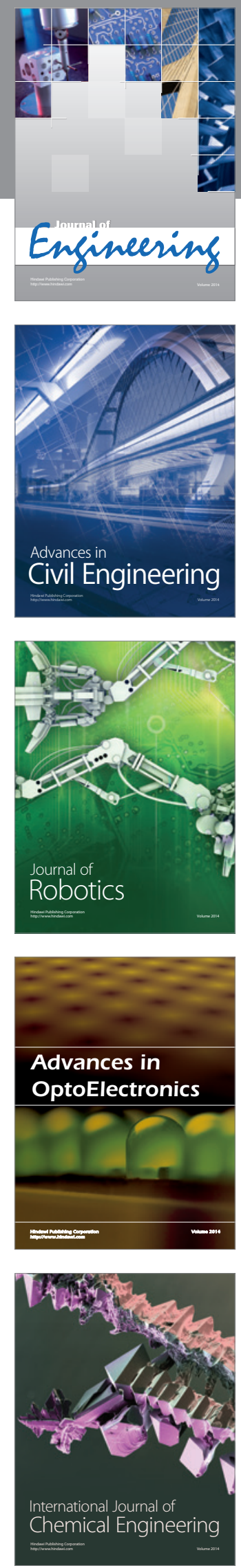

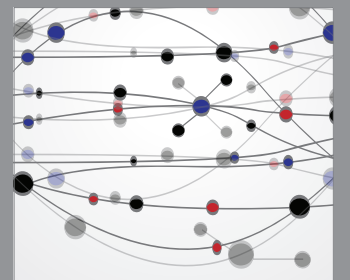

The Scientific World Journal
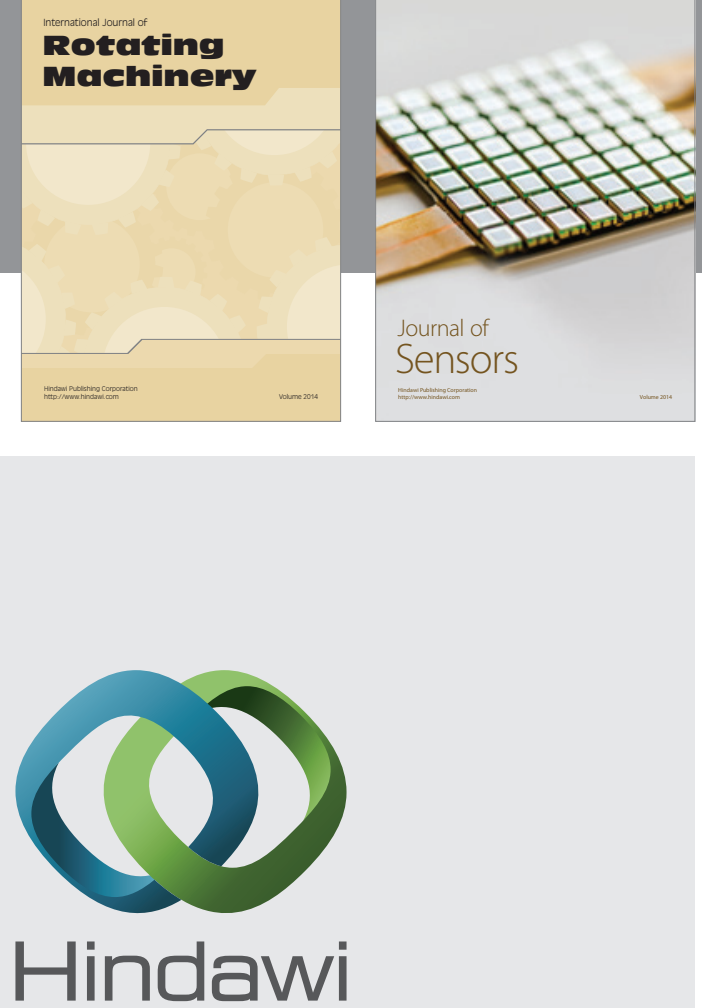

Submit your manuscripts at http://www.hindawi.com
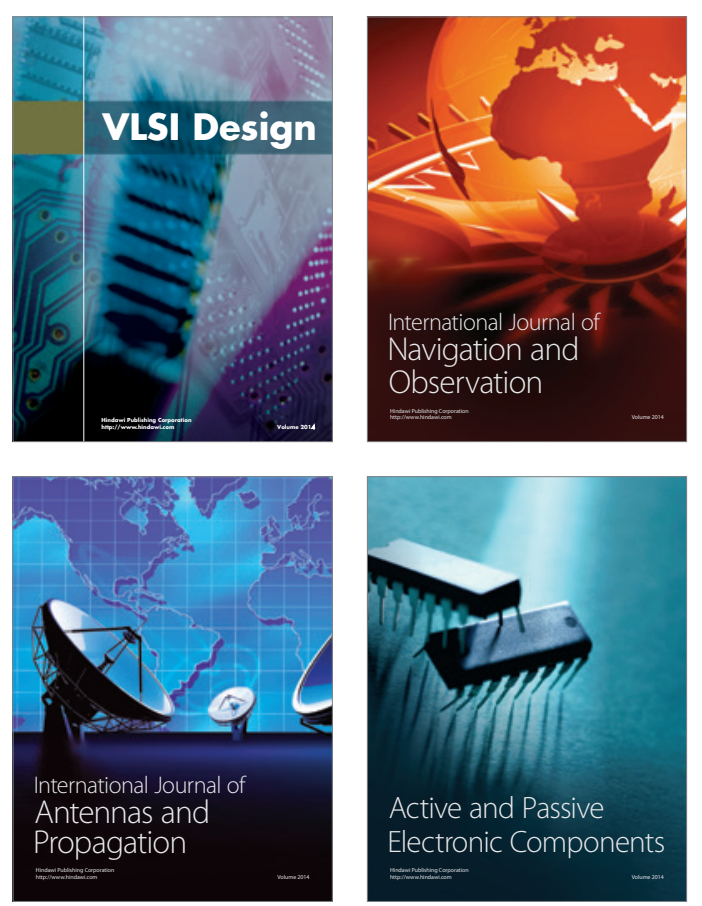
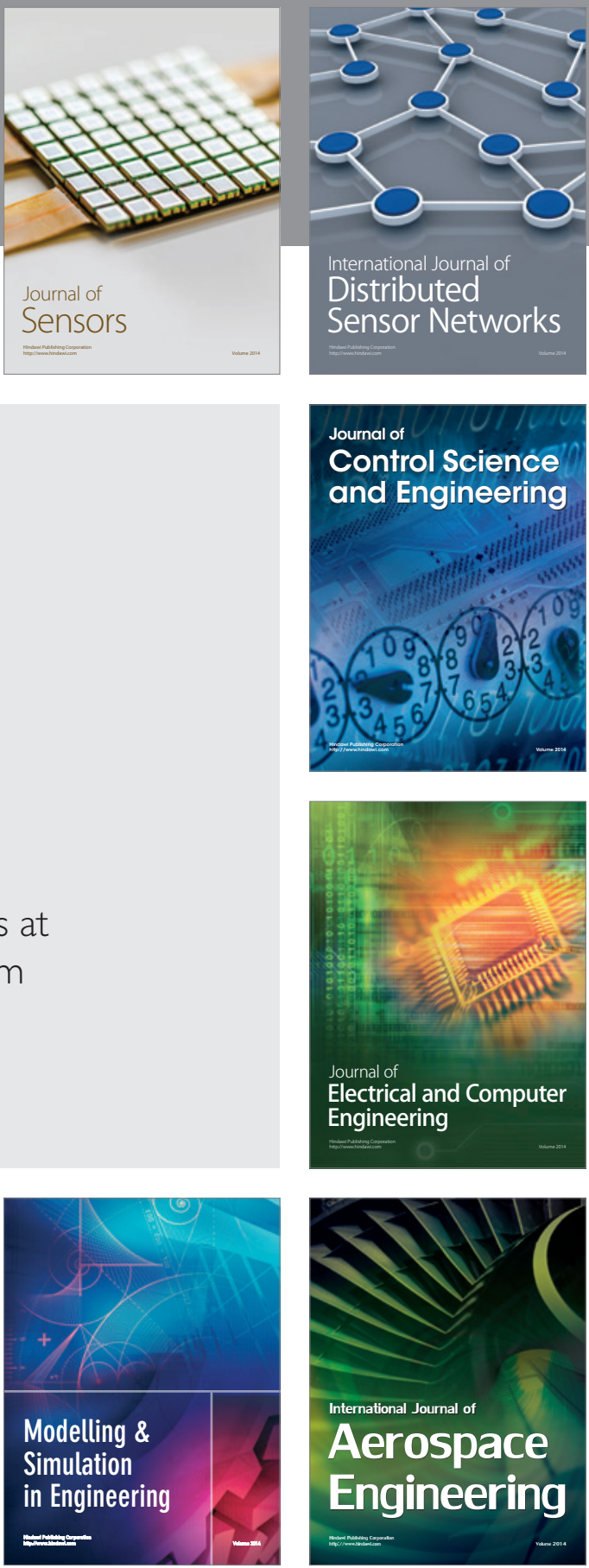

Journal of

Control Science

and Engineering
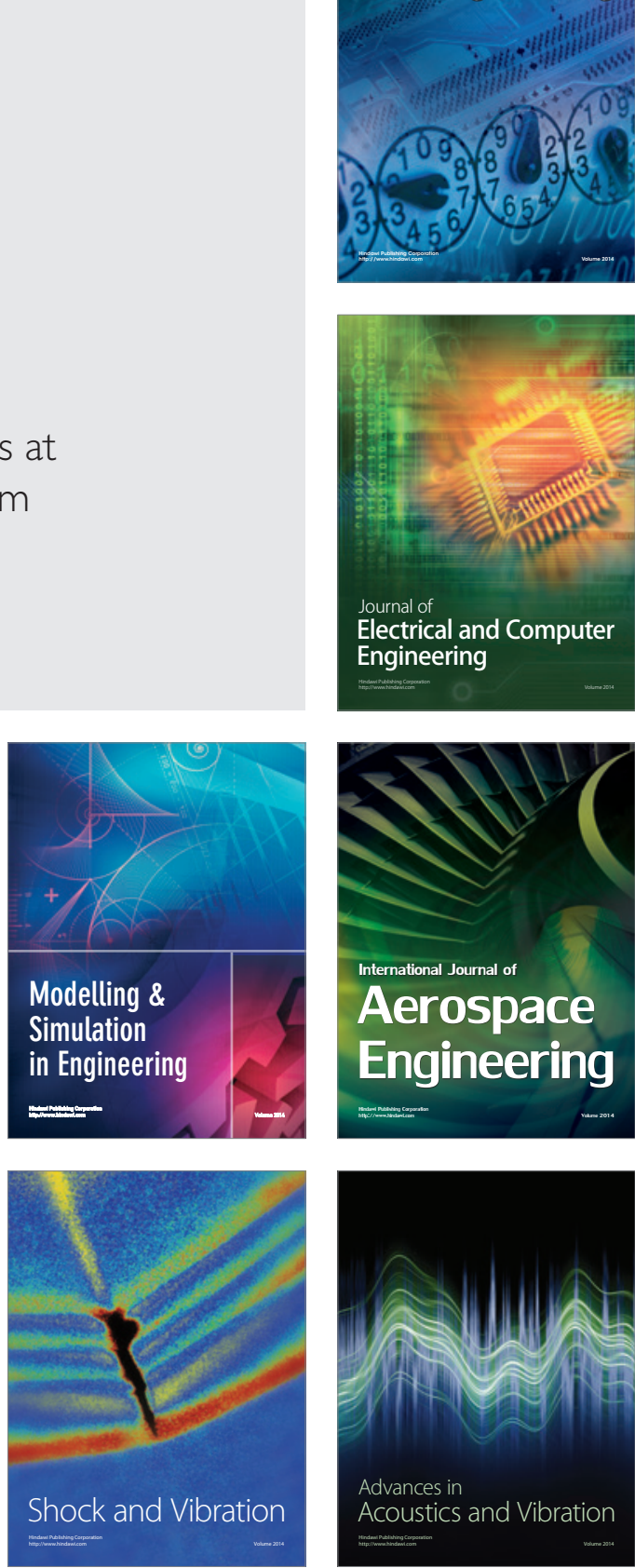